\title{
Um Modelo Matemático da Reinfecção da Rubéola
}

S.M. RAIMUNDO ${ }^{1}$, Faculdade de Medicina da Universidade de São Paulo, FMUSP, Av. Dr. Arnaldo 455, 01246-903 São Paulo, SP, Brasil

H.M. YANG ${ }^{3}$, Departamento de Matemática Aplicada, Instituto de Matemática, Estatística e Computação Científica, UNICAMP, Cx.P. 6065, 13081-970 Campinas, SP, Brasil.

Resumo. Neste trabalho desenvolve-se um modelo matemático que descreve a dinâmica da reinfecção da rubéola e propõe a vacinação como estratégia de controle e/ou erradicação da doença em uma comunidade. Com o objetivo de analisar como a infecção ainda é mantida na população, mesmo existindo um programa de vacinação e revacinação em massa, é feita uma análise qualitativa do modelo determinando seus pontos de equilíbrios e a estabilidade destes pontos.

\section{Introdução}

Com base em publicações de diferentes autores [1],[3],[4],[5], [6] sabe-se que a reinfecção da rubéola pode ocorrer mesmo quando existe imunidade adquirida pela doença ou imunidade induzida pela vacinação. A reinfecção pode ocorrer com ou sem sintomas clínicos e, em qualquer uma destas situações, o número de casos existentes pode ser suficiente para manter o vírus circulando na comunidade. Assim, a possibilidade da reinfecção dos indivíduos que já foram vacinados, ou adquiriram imunidade pela doença, pode ser o veículo pelo qual o vírus continua sendo transmitido e mantido na população.

O esquema proposto pela maioria dos países para a erradicação da rubéola é a imunização seletiva da população, através da vacinação em crianças entre $12 \mathrm{e}$ 15 meses de idade, com a vacina tríplice MMR (sarampo, caxumba e rubéola). Uma dose única da vacina MMR induz a formação de anticorpos contra os três vírus ao menos em $95 \%$ dos suscetíveis vacinados. Entretanto, existem evidências do reaparecimento da rubéola em adultos que foram vacinados de acordo com este esquema e, por esta razão, recomenda-se uma segunda dose entre 3 a 6 anos de idade ou, então, na adolescência. É importante que todos os adolescentes sejam imunes à rubéola, em especial as mulheres que queiram engravidar. A rubéola é geralmente uma doença benigna, mas muito perigosa em mulheres grávidas pois o vírus pode

\footnotetext{
${ }^{1}$ silviamr@dim.fm.usp.br

${ }^{2}$ Centro Universitário Salesiano de São Paulo, Av. Almeida Garret 267, 13087-290 Campinas, SP, Brasil.

${ }^{3}$ hyunyang@ime.unicamp.br
} 
afetar severamente o feto, caracterizando a Síndrome da Rubéola Congênita (SRC) que causa, entre outros, retardo mental, catarata, surdez e problema cardiovascular.

Há poucos anos, a reinfecção era negligenciada pelas autoridades da saúde de todo o mundo mesmo havendo demonstração de que este fenômeno estava ocorrendo. Não existia uma instrução clara para a revacinação de tempos em tempos, a não ser a vacinação na infância. Assim, para avaliar o impacto da reinfecção da rubéola em uma população imunizada pela vacinação, verificava-se a transmissão do vírus em indivíduos imunes e o potencial de risco para uma epidemia ocorrer, sem distinguir a prima infecção das outras infecções. Entretanto, como atualmente já existe uma preocupação com a reinfecção, e alguns países também já estão propondo a revacinação com o objetivo de complementar a imunização, o propósito deste trabalho é formular um modelo no qual a distinção entre a prima infecção e a reinfecção será considerada. Assim, a suposição da vacinação ser aplicada em uma única dose será substituída pelo esquema de vacinação com reforço. Com esta modificação, e com novas hipóteses, a dinâmica do modelo é descrita por sistema de equações diferenciais ordinárias. Com o objetivo de analisar como a infecção ainda é mantida na população, mesmo existindo um programa de vacinação e revacinação em massa para controle ou erradicação da doença, será feita a análise qualitativa do modelo determinando seus pontos de equilíbrios e a estabilidade destes pontos.

\section{Dinâmica da transmissão da rubéola}

O modelo determinístico, que descreve a dinâmica da transmissão da rubéola, considera uma população total $N(t)$ dividida em seis subpopulações. Assume-se que a população total é suficientemente grande para que cada uma das subpopulações possa ser considerada como uma variável contínua, e todos os parâmetros do modelo são positivos. O modelo com dinâmica vital apresenta taxa de mortalidade natural dada por $\mu$ e um fluxo de entrada $\mu N$, positivo e constante, no compartimento de indivíduos suscetíveis. Esses indivíduos tornam-se infecciosos pela "Lei da Ação das Massas em Epidemiologia" (LAME), ou seja, a taxa na qual os indivíduos suscetíveis tornam-se infecciosos é proporcional ao número de encontros entre os suscetíveis e infectados [2]. Define-se $\beta_{1}$, a taxa de transmissão da doença na prima infecção e, $\beta_{2}$, na reinfecção. Os indivíduos recuperam-se a uma taxa $\gamma$, isto é, $\gamma^{-1}$ é o período de tempo no qual os indivíduos permanecem infecciosos, e perdem a imunidade a uma taxa $\rho$, isto é, $\rho^{-1}$ é o período de tempo no qual os indivíduos permanecem imunes à doença.

Dentro deste contexto populacional, a dinâmica da transmissão da doença pode ser descrita. Indivíduos suscetíveis $(S)$ à infecção, ou tornam-se infecciosos $(I)$ e recuperam-se a uma taxa $\gamma$ (caracterizando a prima infecção) ou são vacinados $(V)$ a uma taxa $\nu_{1}$. Os indivíduos vacinados $(V)$, perdendo a imunidade a uma taxa $\rho_{1}$, retornam para uma classe especial de suscetíveis $\left(S^{\prime}\right)$, diferente da primeira, pois já tiveram um contato com o vírus atenuado da doença através da vacina. Nesta classe, os indivíduos suscetíveis tornam-se infecciosos $\left(I^{\prime}\right)$ e recuperam-se a uma taxa $\gamma$. Os indivíduos recuperados ou resistentes a infecção $(R)$ podem também perder a 
imunidade a uma taxa $\rho_{2}$, tornando-se novamente suscetíveis a infecção $\left(S^{\prime}\right)$. Os suscetíveis $\left(S^{\prime}\right)$ podem ser revacinados a uma taxa $\nu_{2}$ ou então tornam-se infecciosos (caracterizando a reinfecção que é responsável pela circulação e manutenção do vírus na população) e recuperam-se a uma taxa $\gamma$. A suposição básica deste estudo é que a população de vacinados $(V)$ são aqueles indivíduos que, embora possuam o registro de vacina na carteira de vacinação, podem perder a imunidade induzida por ela. Por esta razão, considera-se um esquema de vacinação com reforço através do qual os indivíduos são vacinados, com ou sem registro e de forma indiscriminada, para imunizar os indivíduos suscetíveis $\left(S^{\prime}\right)$.

Com base nestas suposições, a dinâmica do modelo pode ser descrita por um sistema de equações diferenciais ordinárias lineares. Como é mais conveniente escrever as equações em termos de frações de indivíduos em cada classe, a dinâmica do modelo será descrita através do seguinte sistema normalizado:

$$
\left\{\begin{array}{l}
\frac{d S}{d t}=\mu-\left(\nu_{1}+\mu\right) S-\beta_{1} S\left(I+I^{\prime}\right) \\
\frac{d V}{d t}=\nu_{1} S-\left(\rho_{1}+\mu\right) V+\nu_{2} S^{\prime} \\
\frac{d I}{d t}=\beta_{1} S\left(I+I^{\prime}\right)-(\gamma+\mu) I \\
\frac{d S^{\prime}}{d t}=\rho_{1} V+\rho_{2} R-\left(\nu_{2}+\mu\right) S^{\prime}-\beta_{2} S^{\prime}\left(I+I^{\prime}\right) \\
\frac{d R}{d t}=\gamma\left(I+I^{\prime}\right)-\left(\rho_{2}+\mu\right) R \\
\frac{d I^{\prime}}{d t}=\beta_{2} S^{\prime}\left(I+I^{\prime}\right)-(\gamma+\mu) I^{\prime},
\end{array}\right.
$$

onde $N(t)=S(t)+V(t)+I(t)+S^{\prime}(t)+R(t)+I^{\prime}(t)=1$.

\section{Análise do modelo}

Com o propósito de identificar se a implantação de uma segunda dose de vacina $\left(\nu_{2}\right)$ é um fator que contribui para o controle e a erradicação da rubéola e que, portanto, deve ser adotada como estratégia em uma comunidade, a análise do modelo (2.1) consiste em determinar seus pontos de equilíbrio e verificar suas condições de existência biológica e de estabilidade.

Inicialmente, é conveniente esclarecer que a estratégia de erradicação proposta pelo modelo (2.1), resultando no equilíbrio trivial, é o esquema de vacinação com dose dupla ou vacinação com reforço: $\nu_{1} \neq 0$ e $\nu_{2} \neq 0$. Assim, a primeira dose é aplicada nas crianças que não possuem o registro na carteira de vacinação, e a segunda, de forma indiscriminada, nos adolescentes. Na seqüência, serão considerados outros dois subcasos do equilíbrio trivial: a população é livre da doença e sem controle, dado por $\left(\nu_{1}=0\right.$ e $\left.\nu_{2}=0\right)$ e a população é vacinada, mas com dose única $\left(\nu_{1} \neq 0\right.$ e $\left.\nu_{2}=0\right)$. 


\subsection{Estratégia de erradicação}

Esquema de vacinação com duas doses ou vacinação com reforço. O sistema (2.1) tem sempre o equilíbrio livre da doença, $P_{o}^{*}=\left(S^{*}, V^{*}, I^{*}, S^{*}, R^{*}, I^{*}\right)=$ $\left(S_{0}, V_{0}, 0, S_{0}^{\prime}, 0,0\right)$, biologicamente viável (todas as coordenadas positivas) dado por

$$
\left\{\begin{aligned}
S_{0} & =\frac{\mu}{\left(\nu_{1}+\mu\right)} \\
V_{0} & =\frac{1}{\left(\rho_{1}+\mu\right)}\left[\frac{\nu_{1} \mu}{\left(\nu_{1}+\mu\right)}+\frac{\rho_{1} \nu_{1} \nu_{2}}{\left(\nu_{1}+\mu\right)\left(\nu_{2}+\rho_{1}+\mu\right)}\right] \\
S_{0}^{\prime} & =\frac{\nu_{1} \rho_{1}}{\left(\nu_{1}+\mu\right)\left(\nu_{2}+\rho_{1}+\mu\right)} .
\end{aligned}\right.
$$

Pela análise dos autovalores do polinômio característico associado à matriz jacobiana do sistema (2.1), o equilíbrio trivial (3.2) é estável quando

$$
\frac{\beta_{1} \mu}{(\gamma+\mu)\left(\nu_{1}+\mu\right)}+\frac{\beta_{2} \nu_{1} \rho_{1}}{(\gamma+\mu)\left(\nu_{1}+\mu\right)\left(\nu_{2}+\rho_{1}+\mu\right)}<1
$$

Da condição (3.3), definindo-se

$$
\begin{array}{ll}
\mathcal{R}_{v}^{1}=\frac{\beta_{1}}{\beta_{1}^{c}}, & \beta_{1}^{c}=\frac{(\gamma+\mu)\left(\nu_{1}+\mu\right)}{\mu} \\
\mathcal{R}_{v}^{2}=\frac{\beta_{2}}{\beta_{2}^{c}}, & \beta_{2}^{c}=\beta_{1}^{c} \frac{\mu\left(\nu_{2}+\rho_{1}+\mu\right)}{\nu_{1} \rho_{1}},
\end{array}
$$

tem-se que o ponto de equilíbrio trivial (3.2) é estável se, e somente se,

$$
\mathcal{R}_{v}^{1}+\mathcal{R}_{v}^{2}<1
$$

Portanto, a condição (3.5) é satisfeita quando $\mathcal{R}_{v}^{1}$ e $\mathcal{R}_{v}^{2}$ são, simultaneamente, menores que um e que somem menos que 1. Para isso, da equação (3.4) observa-se que se o esforço vacinal $\nu_{1}$ for grande, então $\beta_{1}^{c}$ tende para o infinito e $\mathcal{R}_{v}^{1}$ tende para zero. Ainda, mantendo $\nu_{1}$ grande, $\beta_{2}^{c}$ tende para o infinito somente se o esforço vacinal $\nu_{2}$ também for grande, enquanto que $\mathcal{R}_{v}^{2}$ tende para zero. Mais tarde, a condição (3.5) será analisada de forma diferente. Define-se o valor crítico de vacinação $\nu_{1}^{C}$ como função de $\nu_{2}$ e avalia-se qual deve ser o esforço vacinal adotado para se atingir o controle e erradicação da doença.

Esquema sem vacinação. Fazendo $\nu_{1}=0$ e $\nu_{2}=0$ no sistema (2.1), então o novo equilíbrio trivial biologicamente viável, será dado por $P_{o}^{1}=\left(S^{*}, V^{*}, I^{*}, S^{*}, R^{*}, I^{\prime *}\right)=$ $(1,0,0,0,0,0)$, e é estável se, e somente se,

$$
\mathcal{R}_{0}=\frac{\beta_{1}}{(\gamma+\mu)}<1,
$$

isto é, se não existe vacinação, a erradicação da doença é possível somente se a razão de reprodutibilidade basal, $\mathcal{R}_{0}$, for menor que um. Para $\mathcal{R}_{0}>1$, a doença pode ser controlada e erradicada pela vacina, como mostra a equação (3.3).

Esquema de vacinação com dose única ou sem reforço. Fazendo $\nu_{1} \neq 0$ e $\nu_{2}=0$ no sistema (2.1) o novo equilíbrio trivial, biologicamente viável, é dado por $P_{\nu_{1}}=$ $\left(S^{*}, V^{*}, I^{*}, S^{\prime *}, R^{*}, I^{*}\right)=\left(S_{\nu_{1}}, V_{\nu_{1}}, 0, S_{\nu_{1}}^{\prime}, 0,0\right)$, com

$$
\left\{\begin{aligned}
S_{\nu_{1}} & =\frac{\mu}{\left(\nu_{1}+\mu\right)} \\
V_{\nu_{1}} & =\frac{\nu_{1} \mu}{\left(\rho_{1}+\mu\right)\left(\nu_{1}+\mu\right)} \\
S_{\nu_{1}}^{\prime} & =\frac{\nu_{1} \rho_{1}}{\left(\nu_{1}+\mu\right)\left(\rho_{1}+\mu\right)}
\end{aligned}\right.
$$


O ponto (3.7) é estável se, e somente se,

$$
\mathcal{R}_{v_{1}}^{1}+\mathcal{R}_{v_{1}}^{2}<1
$$

com

$$
\begin{array}{lll}
\mathcal{R}_{v_{1}}^{1}=\frac{\beta_{1}}{\beta_{1}^{c}}, & \beta_{1}^{c}=\frac{(\gamma+\mu)\left(\nu_{1}+\mu\right)}{\mu} & e \\
\mathcal{R}_{v_{1}}^{2}=\frac{\beta_{2}}{\beta_{2}^{c}}, & \beta_{2}^{c}=\beta_{1}^{c} \frac{\mu\left(\rho_{1}+\mu\right)}{\nu_{1} \rho_{1}}, &
\end{array}
$$

ou seja, a equação (3.8) é satisfeita quando $\mathcal{R}_{v_{1}}^{1}$ e $\mathcal{R}_{v_{1}}^{2}$ são, simultaneamente, menores que um e que somem menos que 1.

Desde que a equação (3.9) depende do valor de $\nu_{1}$, pode-se rearranjá-la em termos de $\mathcal{R}_{0}$, e obter $\nu_{1}^{C}$, tal que para $\nu_{1} \leq \nu_{1}^{C}$ tem-se $\mathcal{R}_{v_{1}}^{1}+\mathcal{R}_{v_{1}}^{2} \leq 1$.

Assim, da igualdade da equação (3.8), obtém-se o valor crítico de vacinação $\nu_{1}^{C}$ dado por

$$
\nu_{1}^{C}=\frac{\mu\left(\mathcal{R}_{0}-1\right)}{1-\left[\frac{\beta_{2} \rho_{1}}{(\gamma+\mu)\left(\rho_{1}+\mu\right)}\right]} .
$$

Como $\mathcal{R}_{0}>1$, então $\nu_{1}^{C}>0$ se, e somente se,

$$
\beta_{2}<\beta_{2}^{C}=\frac{(\gamma+\mu)\left(\rho_{1}+\mu\right)}{\rho_{1}} .
$$

Da equação (3.11), se $\rho_{1} \rightarrow \infty$, então $\beta_{2}^{C}=(\gamma+\mu)$ e, se $\rho_{1} \rightarrow 0$, então $\beta_{2}^{C} \rightarrow \infty$. Desta forma, conclui-se que a vacinação em dose única só erradica a doença se a reinfecção for fraca $\left(\beta_{2}<\beta_{2}^{C}\right)$. Se a reinfecção for forte $\left(\beta_{2}>\beta_{2}^{C}\right)$, então há necessidade de uma outra forma de controlar a doença, pois a dose única por si só não consegue erradicar.

Além disso, da equação (3.10), também pode-se concluir que a vacinação em dose única erradica a doença se o esforço vacinal $\nu_{1}$ for maior que o seu valor crítico, isto é, $\nu_{1}>\nu_{1}^{C}$. Caso contrário, a doença é mantida na comunidade em níveis baixo. Biologicamente, $\nu_{1}^{C}$ é o esforço vacinal mínimo necessário para diminuir o valor de $\mathcal{R}_{0}$ para um, e, conseqüentemente, o valor de $\mathcal{R}_{v_{1}}^{1}$, tornando possível o controle e a erradicação da doença na comunidade.

Se não existe perda de imunidade pela vacina $\left(\rho_{1}=0\right)$, então $\nu_{1}^{C}=\mu\left(\mathcal{R}_{0}-1\right)$ representa o esforço vacinal mínimo para baixar $\mathcal{R}_{0}$ para valor um, controlando e erradicando a doença na comunidade para $\nu_{1}>\nu_{1}^{C}$. É o modelo $S E I R$, onde $E$ é a classe dos indivíduos expostos ou, o modelo SIR.

A importância da vacina em dose dupla é mostrada pela equação (3.10), pois para $\beta_{2}>\beta_{2}^{C}$, a estratégia de dose única falha. Quando é adotado o esquema com reforço para a erradicação $\left(\nu_{1} \neq 0\right.$ e $\left.\nu_{2} \neq 0\right)$, deve-se observar a condição de estabilidade (3.5). Desta condição, obtém-se o esforço vacinal crítico dependente de $\nu_{2}$ dado por

$$
\nu^{C}\left(\nu_{2}\right)=\frac{\mu\left(\mathcal{R}_{0}-1\right)}{1-\left[\frac{\beta_{2} \rho_{1}}{(\gamma+\mu)\left(\rho_{1}+\nu_{2}+\mu\right)}\right]} .
$$


Observe que $\nu^{C}\left(\nu_{2}\right)$, definido como função de $\nu_{2}$, é positivo se e somente se

$$
\beta_{2}<\beta^{C}\left(\nu_{2}\right)=\frac{(\gamma+\mu)\left(\rho_{1}+\nu_{2}+\mu\right)}{\rho_{1}},
$$

pois $\mathcal{R}_{0}>1$. Se $\rho_{1} \rightarrow \infty$, então $\beta^{C}\left(\nu_{2}\right)=(\gamma+\mu)$ e, se $\rho_{1} \rightarrow 0$, então $\beta^{C}\left(\nu_{2}\right) \rightarrow \infty$.

Observe que a equação (3.10) vem de (3.12), com $\nu_{2}=0$. Assim, $\nu^{C}(0)=\nu_{1}^{C}$. Ainda, a equação (3.11) vem de (3.13), com $\nu_{2}=0$ e $\beta^{C}(0)=\beta_{2}^{C}$.

Análogo ao caso anterior, tomando-se a equação (3.12), pode-se concluir que, se o esforço vacinal for menor que o seu valor crítico, $\nu_{1}<\nu^{C}\left(\nu_{2}\right)$, então a doença se mantém na comunidade. Caso contrário, isto é, se $\nu_{1}>\nu^{C}\left(\nu_{2}\right)$, o controle e a erradicação podem ser alcançados na comunidade. Da equação (3.12) observase que como $\nu^{C}\left(\nu_{2}\right)$ decresce com o aumento de $\nu_{2}$, o esforço $\nu_{1}$ diminui. Dessa forma, o valor de $\mathcal{R}_{v}^{2}$ também diminui. Assim, o controle e a erradicação da doença na comunidade são alcançados. Da equação (3.13), se a reinfecção for forte $\left(\beta_{2}\right.$ crescente) a condição de erradiação sempre é satisfeita se aumentar proporcionalmente $\nu_{2}$. Logo, o esquema de dose dupla não só permite a erradicação da doença, mas contrabalança a perda de imunidade dada por $\rho_{1}$.

\subsection{Estratégias de controle}

Resta agora mostrar que, se não for possível erradicar a rubéola mesmo quando se adota o esquema de vacinação com reforço $\left(\nu_{1} \neq 0\right.$ e $\left.\nu_{2} \neq 0\right)$, então existe um nível endêmico para a doença, representado pelo ponto de equilíbrio não trivial, $P_{e}^{*}=\left(S_{e}^{*}, V_{e}^{*}, I_{e}^{*}, S_{e}^{\prime *}, R_{e}^{*}, I_{e}^{* *}\right)$. Assim, do sistema (2.1), tem-se as coordenadas para $P_{e}^{*}$, obtidas de

$$
\left\{\begin{array}{l}
\mu-\left(\nu_{1}+\mu\right) S_{e}^{*}-(\gamma+\mu) I_{e}^{*}=0 \\
\nu_{1} S_{e}^{*}+\nu_{2} S_{e}^{\prime *}-\left(\rho_{1}+\mu\right) V_{e}^{*}=0 \\
\beta_{1} S_{e}^{*}\left(I_{e}^{*}+I_{e}^{\prime *}\right)-(\gamma+\mu) I_{e}^{*}=0 \\
\rho_{1} V_{e}^{*}+\rho_{2} R_{e}^{*}-\left(\nu_{2}+\mu\right) S_{e}^{\prime *}-(\gamma+\mu) I_{e}^{\prime *}=0 \\
\gamma\left(I_{e}^{*}+I_{e}^{\prime *}\right)-\left(\rho_{2}+\mu\right) R_{e}^{*}=0 \\
\beta_{2} S_{e}^{\prime *}\left(I_{e}^{*}+I_{e}^{*}\right)-(\gamma+\mu) I_{e}^{* *}=0 .
\end{array}\right.
$$

Pode-se de imediato determinar as seguintes coordenadas para o equilíbrio não trivial, $P_{e}^{*}$,

$$
\left\{\begin{array}{l}
S_{e}^{*}=\frac{\mu-(\mu+\gamma) I_{e}^{\prime *}}{\left(\nu_{1}+\mu\right)} \\
V_{e}^{*}=\frac{\nu_{1} S_{e}^{*}+\nu_{2} S_{e}^{\prime *}}{\left(\rho_{1}+\mu\right)} \\
R_{e}^{*}=\frac{\gamma\left(I_{e}^{*}+I_{e}^{\prime *}\right)}{\left(\rho_{2}+\mu\right)}
\end{array}\right.
$$

enquanto que as outras coordenadas são determinadas através das seguintes equações

$$
\left\{\begin{array}{l}
(\mu+\gamma) I_{e}^{*}+\left[-\mu+\frac{(\mu+\gamma)\left(\mu+\nu_{1}\right)}{\beta_{1}}+(\mu+\gamma) I_{e}^{\prime *}\right] I_{e}^{*}-\mu I_{e}^{\prime *}=0 \\
S_{e}^{\prime *} I_{e}^{\prime *}-\left[\frac{(\mu+\gamma)}{\beta_{2}}\right] I_{e}^{\prime *}+S_{e}^{\prime *} I_{e}^{*}=0 \\
\rho_{1} V_{e}^{*}+\rho_{2} R_{e}^{*}-\left(\nu_{2}+\mu\right) S_{e}^{\prime *}-(\gamma+\mu) I_{e}^{\prime *}=0 .
\end{array}\right.
$$


Substituindo-se os valores das coordenadas, dadas por (3.15), na terceira equação de (3.16), determina-se o valor de $S_{e}^{\prime *}$ como função de $I_{e}^{*}$ e $I_{e}^{\prime *}$,

$$
S_{e}^{\prime *}=\frac{\left(\mu+\rho_{1}\right)}{\mu\left(\mu+\nu_{2}+\rho_{1}\right)}\left\{\frac{\rho_{1} \nu_{1} \mu}{\left(\mu+\rho_{1}\right)\left(\mu+\nu_{1}\right)}+k_{1} I_{e}^{*}-k_{2} I_{e}^{* *}\right\}
$$

com

$$
\left\{\begin{array}{l}
k_{1}=\frac{\gamma \rho_{2}}{\left(\mu+\rho_{2}\right)}-\frac{\rho_{1} \nu_{1}(\mu+\gamma)}{\left(\mu+\rho_{1}\right)\left(\mu+\nu_{1}\right)} \\
k_{2}=\frac{\mu\left(\mu+\rho_{2}+\gamma\right)}{\left(\mu+\rho_{2}\right)} .
\end{array}\right.
$$

Resta agora analisar os valores de $I_{e}^{*}$ e de $I_{e}^{\prime *}$ que satisfaçam ao sistema (3.14). Agrupando-se adequadamente os termos da primeira equação de (3.16), obtém-se a seguinte equação para $I_{e}^{*}$,

$$
\left(I_{e}^{*}\right)^{2}+\frac{\mu}{(\mu+\gamma)}\left[\frac{1}{\mathcal{R}_{v_{1}}^{1}}+\frac{(\mu+\gamma)}{\mu} I_{e}^{\prime *}-1\right] I_{e}^{*}-\frac{\mu}{(\mu+\gamma)} I_{e}^{\prime *}=0 .
$$

Tomando

$$
F\left(I_{e}^{\prime *}\right)=\frac{\mu}{(\mu+\gamma)}\left[\frac{1}{\mathcal{R}_{v_{1}}^{1}}+\frac{(\mu+\gamma)}{\mu} I_{e}^{\prime *}-1\right]
$$

e supondo-se que $I_{e}^{* *}>0$, então a equação quadrática (3.18) tem um único valor positivo para $I_{e}^{*}$, independente do valor de $F\left(I_{e}^{* *}\right)$.

Por outro lado, se $I_{e}^{* *}=0$, então, da equação (3.18), tem-se dois casos: $I_{e}^{*}=0$ e $I_{e}^{*} \neq 0$.

Caso 1: Se $I_{e}^{*}=0$ e $I_{e}^{\prime *}=0$, então, da equação (3.17),

$$
S_{e}^{*}=\frac{\nu_{1} \rho_{1}}{\left(\nu_{1}+\mu\right)\left(\nu_{2}+\rho_{1}+\mu\right)}>0 \text {. }
$$

Este caso, analisado anteriormente, corresponde ao equilíbrio trivial dado por $P_{\nu_{1}}=\left(S^{*}, V^{*}, I^{*}, S^{\prime *}, R^{*}, I^{*}\right)=\left(S_{\nu_{1}}, V_{\nu_{1}}, 0, S_{\nu_{1}}^{\prime}, 0,0\right)$ com as coordenadas definidas por (3.7).

Caso 2: Se $I_{e}^{*} \neq 0$ e $I_{e}^{\prime *}=0$, então

$$
I_{e}^{*}=\frac{\mu}{(\mu+\gamma)}\left[1-\frac{1}{\mathcal{R}_{v_{1}}^{1}}\right]
$$

será biologicamente viável, ou seja, $I_{e}^{*}>0 \Leftrightarrow \mathcal{R}_{v_{1}}^{1}>1$.

Assim, quando $I_{e}^{*}>0$ e $I_{e}^{\prime *}=0$,

$$
S_{e}^{\prime *}=\frac{\left(\mu+\rho_{1}\right)}{\mu\left(\mu+\nu_{2}+\rho_{1}\right)}\left\{\frac{\rho_{2} \gamma \mu}{\left(\mu+\rho_{2}\right)(\mu+\gamma)}\left[1-\frac{1}{\mathcal{R}_{v_{1}}^{1}}\right]+\frac{\rho_{1} \nu_{1} \mu}{\left(\mu+\rho_{1}\right)\left(\mu+\nu_{1}\right)} \frac{1}{\mathcal{R}_{v_{1}}^{1}}\right\}
$$

e $S_{e}^{\prime *}>0 \Leftrightarrow \mathcal{R}_{v_{1}}^{1}>1$. 
Entretanto, tem-se que este novo ponto de equilíbrio não trivial dado por $P_{1}=$ $\left(S^{*}, V^{*}, I^{*}, S^{\prime *}, R^{*}, 0\right)$ não é viável, pois para $I_{e}^{\prime *}=0$ e $I_{e}^{*}>0, S_{e}^{\prime *}>0$ não satisfaz a segunda equação de (3.16).

Por fim, substituindo-se o valor da equação (3.17) na segunda equação de (3.16), a seguinte equação quadrática para $I_{e}^{* *}$ é obtida

$$
-k_{3}\left(I_{e}^{\prime *}\right)^{2}+\left[k_{4}-\frac{(\mu+\gamma)}{\beta_{2}}\right] I_{e}^{\prime *}+\frac{\left(\mu+\rho_{1}\right)}{\mu\left(\mu+\nu_{2}+\rho_{1}\right)} k_{5} I_{e}^{*}=0,
$$

onde

$$
\left\{\begin{aligned}
k_{3} & =\frac{\left(\mu+\rho_{1}\right)\left(\mu+\rho_{2}+\gamma\right)}{\left(\mu+\rho_{1}+\nu_{2}\right)\left(\mu+\rho_{2}\right)} \\
k_{4} & =\frac{\left(\mu+\rho_{1}\right)}{\mu\left(\mu+\nu_{2}+\rho_{1}\right)}\left\{\frac{\mu \rho_{1} \nu_{1}}{\left(\mu+\rho_{1}\right)\left(\mu+\nu_{1}\right)}+\left(K_{1}+K_{2}\right) I_{e}^{*}\right\} \\
k_{5} & =\frac{\rho_{1} \nu_{1} \mu}{\left(\mu+\rho_{1}\right)\left(\mu+\nu_{1}\right)}+K_{1} I_{e}^{*} .
\end{aligned}\right.
$$

Analogamente ao caso anterior, definindo

$$
G\left(\beta_{2}\right)=k_{4}-\frac{(\mu+\gamma)}{\beta_{2}},
$$

observa-se que existe um único valor positivo para $I_{e}^{\prime *}$, independente do valor de $G\left(\beta_{2}\right)$

Além disso, se $I_{e}^{*}=0$, então, da equação quadrática (3.19), tem-se novamente dois casos: $I_{e}^{\prime *}=0$ e $I_{e}^{\prime *} \neq 0$.

Caso 1: Para $I_{e}^{\prime *}=0$ e $I_{e}^{\prime *}=0$, já foi analisado para a equação quadrática (3.18).

Caso 2: Para $I_{e}^{\prime *}=0$, então

$$
I_{e}^{\prime *}=\frac{\left(\mu+\rho_{1}+\nu_{2}\right)\left(\mu+\rho_{2}\right)(\mu+\gamma)}{\left(\mu+\rho_{1}\right)\left(\mu+\gamma+\rho_{2}\right) \beta_{2}}\left\{\mathcal{R}_{v}^{2}-1\right\}
$$

será biologicamente viável $\left(I_{e}^{\prime *}>0\right)$ se, e somente se, $\mathcal{R}_{v}^{2}>1$. Observando que, da equação quadrática para $I_{e}^{*}$, tem-se que

$$
\frac{-\mu}{(\mu+\gamma)} I_{e}^{\prime *}=0
$$

conclui-se que este caso também não e viável.

Assim, para o sistema (2.1), se existe um ponto de equilíbrio não trivial e biologicamente viável $P_{e}^{*}=\left(S_{e}^{*}, V_{e}^{*}, I_{e}^{*}, S_{e}^{\prime *}, R_{e}^{*}, I_{e}^{\prime *}\right)$, então ele é único.

Sob o aspecto biológico isto significa que, quando se adota o esquema de vacinação em duas doses e não se alcança a erradicação da doença, então pode-se controlar o aumento no número de casos da doença, evitando-se assim um surto epidêmico. Assim, se a condição (3.5) não é satisfeita e a soma dos valores para $\mathcal{R}_{v}^{1}$ e para $\mathcal{R}_{v}^{2}$ é maior que um, existe um único nível endêmico para a doença na comunidade. 


\section{Conclusão}

A proposta deste trabalho foi desenvolver um modelo para descrever a dinâmica da transmissão da rubéola quando se adota duas doses da vacina como estratégia de erradicação ou controle: uma, o MMR (sarampo, cachumba e rubéola) em crianças que não possuem registro na carteira de vacinação, a outra, de forma indiscriminada, na adolescência.

Através do estudo analítico do modelo foi possível concluir que, existindo vacinação, a doença pode ser erradicada mesmo quando a razão de reprodutibilidade basal for $\mathcal{R}_{0}>1$. No caso de existir apenas uma única dose vacinal, então para a erradicação ser alcançada é necessário que o esforço vacinal seja maior que o seu valor crítico $v^{C}$, e a reinfecção seja fraca $\left(\beta_{2}\right)$.

Por outro lado, se o esquema de vacinação com reforço ou com duas doses é adotado, então a erradicação é sempre possivel ainda quando o esforço vacinal relativo à primeira dose for pequeno. Para isso, é suficiente aumentar o esforço vacinal na segunda dose. Vale ressaltar o cuidado que se deve ter neste caso para se atingir o objetivo de controle e erradicação: para diminuir o esforço $v_{1}$, quanto deve-se aumentar o esforço na segunda dose? É necessário avaliar a diminuição e o aumento dos esforços para que não sejam feitos esforços a menos na primeira dose e esforços exagerados na segunda.

Além disso, mostrou-se que existe um único equilíbrio não trivial, que é biologicamente viável, quando $\mathcal{R}_{v_{1}}^{1}>1$ e $\mathcal{R}_{v_{1}}^{2}>1$. Ou seja, se o controle e a erradicação não forem alcançados, ainda assim pode ser possível evitar uma epidemia quando se adota como estratégia o esquema de vacinação com reforço.

E, muito embora o modelo tenha sido bastante simples sob o ponto de vista matemático, o resultado epidemiológico mostrou que um modelo matemático pode ser um instrumento importante para fornecer subsídios quanto a mecanismos de controle de doenças na comunidade.

Abstract. In this paper a mathematical model to describe the dynamics of rubella infection is proposed. The model deals with the assumption that two vaccination schedules are applied into community. The analytical study of the model shows that this kind of control strategy can eradicate the rubella in a community depending on the vaccination programme.

\section{Referências}

[1] J.E. Cradock-Watson, M.K.S. Ridehalgh, M.J. Anderson e J.R. Pattison, Rubella reinfection and the fetus, The Lancet, 1039, (1985).

[2] W.H. Hamer, Epidemic Disease in England, The Lancet, (1906), 733-739.

[3] H.M. Yang, Mathematical Modeling of the Infectious Diseases considering the Re-infection and the vaccine failures, em "Mathematical Modelling \& Computing in Biology and Medicine", ESMTB $5^{\text {th }}$ Conference, Milano, Italy, 2002. 
[4] E. Massad, M.N. Burattini, R.S. Azevedo-Neto, H.M. Yang, D.M.T. Zanetta e F.A.B. Coutinho, A model-based design of a vaccination strategy against rubella in a non-immunized community of São Paulo State, Brazil, Epidemiol. Infect., 112 (1994), 579-594.

[5] P. Morgan-Capner, C. Burgess, R.M. Ireland e J.C. Sharp, Clinically apparent rubella reinfection with a detectable rubella specific IgM response, $B M J, 286$ (1983), 1616.

[6] S.M. Raimundo, E. Massad e R.S. Azevedo Neto, The impact of reinfection on the control of rubella: a mathematical model approach, em "Abstracts of the 8th International Congress on Infectious Diseases", p. 212, Boston, Massachusets, U.S.A., May, 1998. 